\title{
Supporting Young Adults with Autism Spectrum Disorders (ASD) to Maximise Their Potential
}

\author{
Satheesh Gangadharan ${ }^{1} \cdot$ Sabyasachi Bhaumik $^{1} \cdot$ Rohit Gumber $^{1}$
}

Published online: 28 April 2016

(C) Springer India Pvt. Ltd. 2016

\section{Introduction}

There has been a significant increase in the number of individuals diagnosed as having an Autism Spectrum Disorder (ASD). A large scale American Survey of children showed that the prevalence of ASD increased by $75 \%$ from 2002 to 2008 [1]. ASD is a complex neurodevelopmental disorder which tends to have a profound impact not only on individuals affected by the condition, but also on people caring for them. Many family carers seek help from a range of professionals/services to improve the skills of the person they look after, with a hope that the individual has a more fulfilling life. The individuals with ASD face challenges in many fronts starting from childhood but transition from childhood to adulthood is one of the most challenging times. Many would struggle to cope unless it is handled carefully with good planning.

Effective transition planning is vital in ensuring that the individual is given the optimal opportunity for the future. This should start sufficiently in advance while the young person is in school. If young adults with ASD do not transit directly into employment, they have a much lower chance of getting a gainful employment. The good transition plan would involve the following.

\section{Person Centred Planning}

Person-centered planning (PCP) is a well-known collaborative framework that uses principles of involvement of the

Satheesh Gangadharan satminiuk@gmail.com

1 Psychiatry of Intellectual Disability, Leicestershire Partnership NHS Trust, Groby Road, Leicester LE2 4UD, UK individual with ASD and people in the circle of care to produce a plan that truly reflects the individual's aspirations and strengths. A variety of tools are available to develop a good PCP including Making Action Plans (MAPS), Group Action Planning (GAP) and Planning alternative tomorrow with hope (PATH) [2]. The PCP when used for transition planning keep the focus on the individual's interests, choices, communication and other support needs. The aim of transition planning would be to provide the support that is needed for the individual to cope with the change, make the right choices and keep a sense of control of the process.

\section{Further Education}

Most individuals with ASD would need further education after school years focusing on practical skills required as adults which could range from training technical skills required for a job, social skills training, managing finance or skills required for day to day living such as cooking, maintaining personal hygiene etc. For those individuals with focused special interests, these interests could be utilised to develop skills that are useful for employment if appropriate. It is important to remember that approximately two-thirds of individuals with ASD may have associated intellectual disability [3] and these individuals, depending on their ability levels may still be gainfully occupied provided they have the right support. Ongoing support is essential in applying the skills in the different facets of their adult life as people with ASD may have difficulty generalising the learning.

\section{Optimising the Prospect for Employability}

Historically people with ASD had limited chance of employment which is now changing. At the same time 
people with ASD value the prospect of employment which gives them enormous satisfaction, rather than attending day care activities or being at home. Increasing the chance of employability would include

a. Vocational training early on in school and in Further Education: Vocational training should be based on the strengths of the individual but matched with the overall abilities of the individual. A good vocational training could be used in adapting special interests which are repetitive and non-functional into a passion which has deep meaning for the individual and useful for employment purpose. Vocational training also needs to include the ability to develop attention to tasks with less supervision; completion of tasks at a pace satisfactorily as well as with good quality, ability to wait for one's turns and maintaining personal appearance and hygiene.

b. Comprehensive assessment of support needed for employment: The assessment should include

1. Support needed for communication in the work environment.

2. The extent of peer support and mentoring required.

3. Nature of the physical environment that would maximise the success potential such as the level of sensory stimuli (noise level, lighting etc.); availability of space to have breaks etc.

4. Structure, routine and predictability of work schedule.

5. Ongoing training and prompts required in the employment context.

6. Level of independence such as travelling independently, emotional regulation, personal safety, handling money, social engagement; training required to develop community awareness.

7. Factors that helps the person cope with difficult situations and support required to manage crisis situations.

c. Availability of Autism friendly employers: employers who see the benefits of individuals with ASD as employees are likely to be more supportive. People with ASD for example are likely to very punctual, less distracted by social distractions in work place and are likely to be productive in a structured environment. The following are some of the measures that could help an individual with ASD in a work environment.

1. Improving the awareness of ASD among coworkers.

2. Specific person who can provide one to one assistance like a mentor, buddy or a champion.

3. Prompts or cues to perform job tasks.

4. Regular breaks during work.

5. Assistance with time management.
6. Alternative choices for behaviour.

7. Altering physical environment to minimise distraction.

\section{Enhancing the Communication Support}

Individuals with ASD have variable levels of communication difficulties. While most children with ASD will experience significant delay in development of language, later in life some will develop sufficient language skills to communicate. However even those with good vocabulary may have difficulty in undertaking reciprocal conversations as well as follow nuances of social communications. A significant number of people with ASD would have difficulty in communicating verbally. A variety of communication supports could be used to augment the communication potential of individuals including Picture Exchange Communication System (PECS); sign languages as well as computer assistive technology [4]. A thorough assessment of an individual's communication needs and use of appropriate support will greatly enhance the potential of the individual as well as minimise setbacks. We are aware that, in many countries availability of professionals, especially Speech and Language therapists, is limited. This can be mitigated to some extent by improving awareness of teachers, further education staff as well as family on the communication needs of individuals with ASD as well as the use of appropriate assistive technology.

\section{Management of Behaviours}

Successful integration of adults with ASD into an environment would depend on the ability to self regulate own behaviour. However the maladaptive behaviours are likely to be less if the environment is adapted to the needs of the individual. The maladaptive behaviours include repetitive behaviour which affects the day-to-day functions including work, social withdrawal, lack of engagement with activities, self-injurious or severe selfstimulation behaviour and aggression to people or property. Behaviour in individuals with ASD may have multiple functions. The function of the behaviour could be communication of distress, response to sensory problems (usually a form of sensory stimulation such as bright light, noise etc.), the result of an increasing anxiety or a physical discomfort such as pain. The effective management of behaviour would depend on identification of the function of the behaviour and intervening to manage it. Use of behavioral observations and functional analysis followed by appropriate management using behavioral principles would reduce the behaviour. 


\section{Diagnosis and Management of Mental Health or Physical Health Problems}

Individuals with ASD are vulnerable to develop mental health problems such as anxiety, depression, attention deficit hyperactivity disorder etc. Often the presentation of mental health problem can be atypical and therefore missed. There is also an issue of diagnostic overshadowing; attributing the symptoms to underlying ASD rather than the co-existing mental disorder. One study in children found that $70 \%$ percent of participants had at least one co-morbid disorder and $41 \%$ had two or more [5].

Individuals with ASD has a higher prevalence of anxiety than the general population. Parents report high prevalence of anxiety in young people with autism which can exacerbate social deficits, impair daily living skills and impact on relationships with peers and family. While to a great extent anxiety in people with ASD can be managed with structured and supportive environment, good support for communication and predictability, some individuals with ASD would have high level of anxiety that would need psychological or pharmacological interventions. There is some evidence on the use of Cognitive Behavioural Therapy for individuals with high functioning autism [6]. Use of medications like Citalopram from the Serotonin Re-uptake inhibitor group also has been reported to have some effect in reducing the intensity of anxiety [7].

Individuals with ASD also have a high prevalence of certain physical health problems such as epilepsy [8]. Epilepsy persists into adult life with remission only in $16 \%$. It is essential that this would need early diagnosis, good treatment which includes the correct anti-epileptic medications, use of appropriate rescue medication in those with refractory epilepsy resulting in prolonged seizures as well as education of the individual with ASD and people in the circle of care about the safety precautions and support. Lack of adequate support for epilepsy can significantly restrict an individual's ability to realise his or her potential.

\section{Active Involvement and Support of Family and People in the Circle of Care of the Individual with ASD}

Immediate family play a pivotal role in supporting young people with autism. Mothers with an adolescent with autism report lower level of well being compared to other developmental disorders. In addition, many siblings have felt that their parents perceived their needs as being secondary, with more time and attention given to the child with autism [9]. Developing strategies that are family centered and encourage active participation of the whole family would therefore be ideal. Families would need professionals to work with them in helping them understand the problem and shown them how best to support individuals with ASD. Managing difficult behaviour, ensuring the safety of the individual and availability of educational, occupational and health support are the key concerns of families in an Indian Context. It is important to acknowledge that on some occasions parents by virtue of their experience may have more expertise on the nature of problem and the support required than the professionals. It is therefore important that professionals working in this area form an effective partnership approach to support the individual where the family is seen as an equal partner. Many parents find it useful to talk to other parents who face similar situations. Establishing support groups for the family carers is a good way of enabling families to learn from each other and problem solve.

\section{Conclusion}

Despite the increasing prevalence of people diagnosed with ASD, the support services for individuals with ASD are patchy and isolated. There are even more limited opportunities for occupational skills developments, employment guidance or support for securing employment. Successful integration of people with ASD in the community needs an approach that enables the individuals with ASD to develop skills required for useful employment as well as independent or semi-independent living in the community. Good team working is needed between the professionals and family carers to support the transition of people with ASD into adulthood. It is equally important to create opportunities for individuals with ASD with better support for occupational skills development, job coaching as well as autism-friendly employers. The way ahead therefore will be through empowerment of individuals with ASD and their family carers, policy changes that enhance educational and employment support, establishment of parental support groups, and improving the awareness of the needs of young adults with ASD for professionals like paediatricians, psychiatrists, psychologists, occupational therapists and speech and language therapists as well as educational staff.

\section{References}

1. Prevalence of Autism Spectrum Disorders (ASDs), among multiple areas of the United States in 2008. The Centres for Disease Control and Prevention (CDC), U.S. Department of Health and Human Services. http://www.cdc.gov/ncbddd/autism/documents/ ADDM-2012-Community-Report.pdf

2. Amado AN, McBride M. Increasing person-centered thinking: improving the quality of person-centered planning: a manual for 
person-centered planning facilitators. Minneapolis, MN: University of Minnesota, Institute on Community Integration; 2001.

3. Dykens EM, Lense M. Intellectual disabilities and autism spectrum disorder: a cautionary note. In: Amaral D, Dawson G, Geschwind D, editors. Autism spectrum disorders. New York: Oxford University Press; 2011. p. 261-9.

4. Bondy A, Frost L. A Picture's Worth: PECS and Other Visual Communication Strategies in Autism. Topics in Autism; Woodbine house, 6510 Bells Mill Rd., Bethesda,MD 20817,info@woodbinehouse.com; http://www.woodbine.com.

5. Simonoff E, Pickles A, Charman T, Chandler S, Loucas T, Baird G. Psychiatric disorders in children with autism spectrum disorders: prevalence, comorbidity, and associated factors in a population-derived sample. J Am Acad Child Adolesc Psychiatry. 2008;47(8):921-9.
6. Drahota A, Wood JJ, Sze K, Van Dyke M. Effects of cognitive behavioral therapy on DLS in children with high-functioning autism and concurrent anxiety disorders. J Autism Dev Disord. 2011;41:257-65.

7. Vasa RA, Carroll LM, Nozzolillo AA, Mahajan R, Mazurek MO, Bennett AE, Bernal MP. A systematic review of treatments for anxiety in youth with autism spectrum disorders. J Autism Dev Disord. 2014;44(12):3215-29.

8. Danielsson S, Gillberg IC, Billstedt E, Gillberg C, Olsson I. Epilepsy in young adults with autism: a prospective populationbased follow-up study of 120 individuals diagnosed in childhood. Epilepsia. 2005;46:918-23.

9. Gupta A, Singhal N. Psychosocial support for families of children with autism. Asia Pac Disabl Rehabl J. 2005;16(2):62-83. 